\title{
Impact Damage Management of Composite Laminated Structures by a Probabilistic Approach
}

\author{
A. Riccio ${ }^{*}$, T. Russo and F. Scaramuzzino
}

Second University of Naples, Department of Industrial and Information Engineering, via Roma $n$ 29, 81031 Aversa, Italy

\begin{abstract}
A numerical procedure for the design of impact damage resistant composite laminated structures is proposed in the present paper. This procedure, based on probabilistic analyses, is able to support the design of complex composite laminated structures by accounting for the scatter in composite materials' mechanical properties (related to the mutable environmental conditions experienced by an aircraft component during its service life) and by accounting for the uncertainty on accidental impacts locations. A failure criterion, based on the determination of the threshold critical impact energy for impact induced delaminations' onset, is adopted for the evaluation of the damage resistance of composite laminated structures, in the frame of the introduced probabilistic analyses.

The proposed procedure has been implemented into a commercial FEM platform and composite panels have been used as numerical benchmarks to investigate the influence of the combined material properties and impact location uncertainties on composite laminated structures' impact damage resistance. A further comparison between two different stiffened composite panels designs have been introduced to highlight the added value of the proposed impact damage resistance probabilistic approach in terms of safety and effectiveness, with respect to the standard deterministic tools.
\end{abstract}

Keywords: Probabilistic design, impact, delamination, FEM, composites.

\section{INTRODUCTION}

It is known that the low velocity impact phenomenon represents one of the most common sources of damage in composite laminated structures. Low velocity impacts with foreign objects can occur accidentally and, usually, can involve different interacting failure mechanisms such as matrix cracking, fibre cracking, delamination and indentation [1-3]. The study presented in this paper, is focused on impact induced delaminations which can cause a strong structural stiffness and strength reduction. Additionally, these failure mechanisms can be hardly detected by visual inspections, because, especially in thin composite laminates, low velocity impact induced delaminations can arise deep in the laminate and far from the external surface where the impact event takes place [4-6].

When designing aeronautical composite components, which are exposed to impact threats, the effect of impact induced delamination must be taken into account. The damage management approach commonly adopted in aerospace is based on an impact damage tolerance design philosophy, based on material allowable reduction. According to this design philosophy, to face the impact events, a structure must be designed capable to withstand the service loads even if subjected to multiple impact events inducing multiple delaminations [7].

*Address correspondence to this author at the Second University of Naples, Department of Industrial and Information Engineering, via Roma n 29, 81031 Aversa, Italy; Tel: +39 081 5010504; Fax: +39 081 5010204;

E-mail: aniello.riccio@unina2.it
An alternative damage management approach, currently studied in the frame of several research projects, is based on an impact damage resistance philosophy according to which a composite structural component must be designed capable to totally resist (without developing any significant damage) the impact events which can take place during the service life $[8,9]$.

Focusing on impact induced delaminations, the damage resistance of impacted composite laminated structures can be predicted by evaluating the "threshold critical impact energy" needed for the onset of impact induced delaminations [10-12]. According to this approach, if the impact energy is below the threshold level, the impact does not cause delaminations in the laminate. On the contrary, when the impact energy is just above this value, the delaminations are considered to take place instantaneously.

As known, due to changes in environmental conditions, composites components can be characterized by significant material properties variations during their service life [13, 14]; these variations can have relevant effects on the damage mechanisms of composites [15] and, in particular, on delaminations [16]. Impact induced delamination onset and evolution, can also be influenced by the impact location, especially in complex composite laminated structures characterized by a non-uniform material distribution and by relevant geometrical discontinuities (stiffened panels, etc.) [17].

In this paper a study on the influence of the scatter of material mechanical properties, and of the randomness of the impact location, on composite panels impact damage 
resistance, is presented. To take into account these sources of variability, a probabilistic approach is introduced being able to accept the input parameters as stochastic functions. The study presented in this paper can be considered as the natural extension of the research activity introduced in [18] dealing with the investigation of the influence of material properties uncertainty on the damage resistance of composite panels (without considering the variability of the impact location).

As a matter of fact, the introduced probabilistic approach, by means of Monte Carlo based Simulations and adopting the threshold critical impact energy approach $[10,12]$ for the assessment of the impact induced delaminations, is able to take into account simultaneously the scatter of material mechanical properties, and the randomness of the impact location when determining the impact damage resistance of composite laminated structures. The material properties stochastic distributions, used for the numerical simulations, have been taken from an experimental program, on a number of laminate samples respecting specific conditions of temperature and moisture levels.

Three numerical applications are introduced to demonstrate the effectiveness of the introduced approach. The first numerical application on a flat composite panel is aimed to quantify the influence of material properties scatter on the impact damage resistance. Results in terms of cumulative distribution functions of output variables related to the damage resistance are presented and critically assessed, providing information about the risk to miss the damage resistance requirements.

A further numerical application on a stiffened composite panel considers the variability of mechanical properties, due to environmental effects, and the randomness of the impact location. For both these applications, some indications on the contribution of each design parameter to the overall risk to miss the damage resistance requirements are also provided.

The last numerical application is aimed to compare the stochastically assessed impact damage resistance behavior of two stiffened composite panels. A criterion to establish the best performance in terms of stochastically assessed impact damage resistance is suggested. A further comparison with deterministic results is used to demonstrate the real added value of the proposed probabilistic approach in terms of effectiveness and reliability of composite laminated structures impact damage resistance evaluation.

In section 2 the theory behind the proposed probabilistic approach is detailed while in section 3 the first two numerical applications, finalized to the investigation of the influence of the material properties and impact location uncertainties on the impact damage resistance evaluation, are presented. Finally, in section 4 , the last numerical application, finalized to the demonstration of the effectiveness of the proposed probabilistic approach for the impact damage resistance assessment, with respect to standard deterministic approaches, is shown.

\section{THEORY BEHIND THE PROPOSED PROBABILI- STIC APPROACH}

As already mentioned, the capability of a composite structure to fulfil the damage resistance requirements can depend on two main sources of variability linked to the scatter of material mechanical properties and to the randomness of the impact location. In order to take into account the influence of these uncertainties on impact induced delaminations occurrence, the probabilistic procedure, schematically described in Fig. (1), has been implemented in the Probabilistic Design System (PDS) of the FEM code Ansys [19].

According to the schematic representation of Fig. (1), deterministic (geometry, material lay-up, impact force, boundary conditions) and probabilistic input variables (material properties and impact location) are defined preliminary. The probabilistic system PDS, using the Monte Carlo's method [20], chooses a set of samples defined by the deterministic variables and by values of the probabilistic variables selected from proper stochastic distribution functions. The probabilistic module calls the Davies' deterministic procedure $[10,12]$, implemented as Ansys Design Parametric Language (APDL) script in ANSYS, which, together with a static linear analysis, is used to evaluate the impact damage resistance. This calculation is repeated for each different set of probabilistic variables values sampled from the statistical distribution functions of the input parameters according to the chosen sampling method. The statistical distributions of the threshold load and energy levels inducing delamination onset (output of the probabilistic procedure) will give us a measure of the impact damage resistance of the analyzed composite configurations. In the following subsections, the Monte Carlo simulation method and the Davies procedure, adopted in the frame of the probabilistic procedure are briefly introduced.

\subsection{The Monte Carlo Simulation Method}

The Monte Carlo Simulation method is the most common and traditional method for a probabilistic analysis, especially for aerospace applications [21]. It is a stochastic method where the inputs are randomly generated from probabilistic distribution functions in order to simulate the process of sampling from an actual population. For this method it is possible to employ either the random Sampling approach (Direct Monte Carlo Simulation) or the Latin Hypercube Sampling approach $[22,23]$.

The random sampling technique is the most common and traditional form of sampling in a Monte Carlo simulation even if it is not the most efficient one. However, it is still widely used and accepted, especially for benchmarking and validating probabilistic results. The inefficiency of the random sampling approach is related to the huge number of simulation loops required and to the lack of "memory" in the sampling process (sampling points can be close to each other).

This kind of sampling approach, although could lead to inefficient sampling, well describes the natural intrinsic variability of the random parameters.

The inefficiency of the random sampling approach can be avoided by means of the Latin Hypercube Sampling (LHS) technique, which, unlike the Random Sampling one, has a sample "memory" and avoids the duplication of samples that have been evaluated before by portioning the analysed domain in sub-domains of equal probability content and choosing only one sample point per sub-domain (usually in a random location or in the center of the sub-domain). As a 


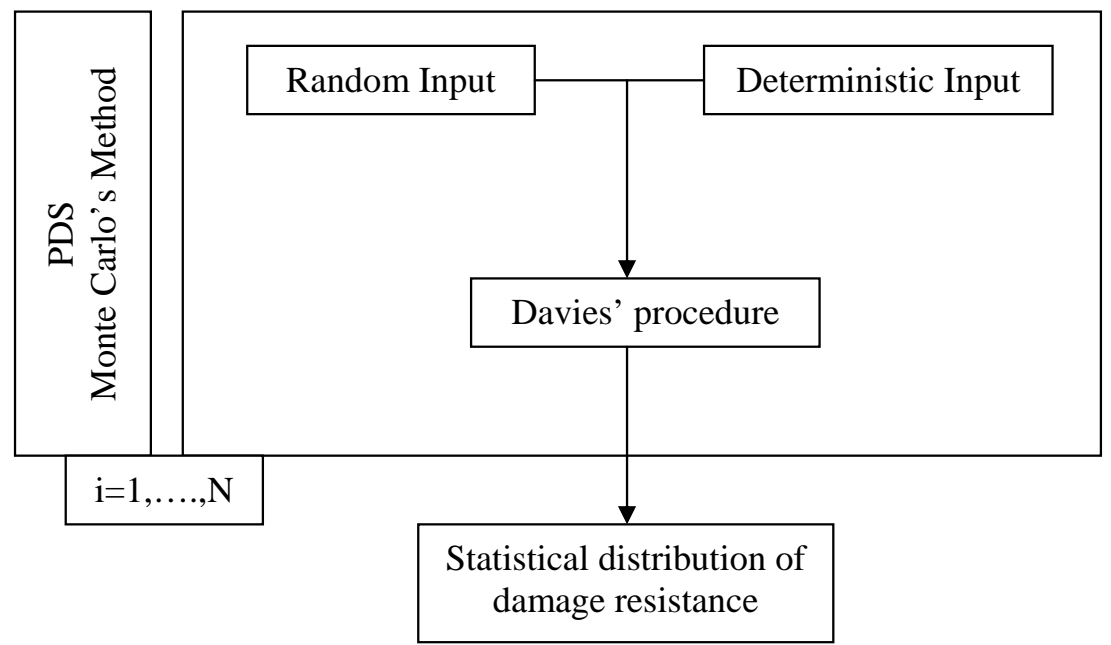

Fig. (1). Schematic representation of the probabilistic procedure.

matter of fact, in the context of statistical sampling, a square grid containing sample positions is a Latin square if (and only if) there is only one sample in each row and each column. A Latin hypercube is the generalization of this concept to an arbitrary number of dimensions whereby each sample is the only one in each axis-aligned hyperplane containing it. Generally, with the LSH technique, the extremities of a distribution are forced to participate in the sampling process.

In Fig. (2), a schematic representation of sample distributions obtained by means of the random sampling approach (Fig. 2a) and the Lain Hypercube Sampling approach (Fig. 2b), are presented.

The Latin Hypercube Sampling technique requires, usually, $20 \%$ to $40 \%$ fewer simulations loops than the random sampling technique to provide the same results with the same accuracy. The maximum number of combinations for a Latin Hypercube of $\mathrm{M}$ divisions and $\mathrm{N}$ variables (i.e., dimensions) can be computed according to the following relation:

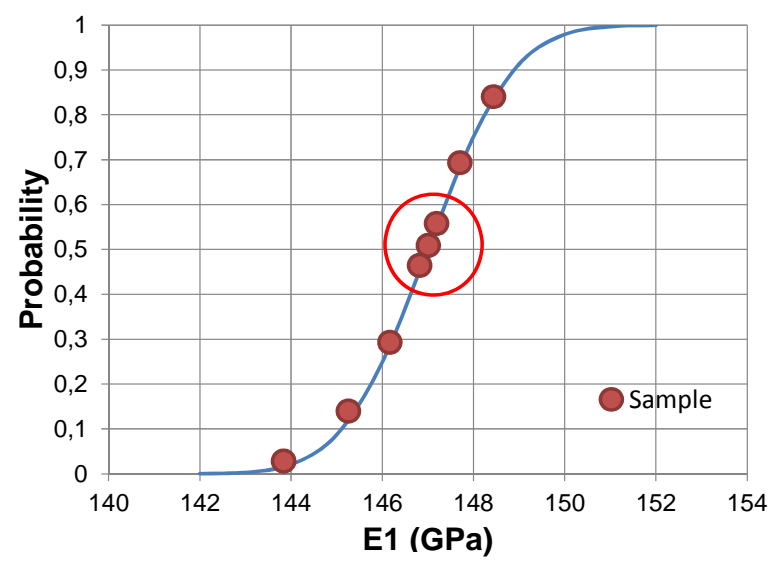

a)

$$
\prod_{n=0}^{M-1}(M-n)^{N-1}=(M)^{N-1}
$$

\subsection{Evaluation of the Impact Damage Resistance}

The approach introduced in $[10,12]$, has been implemented in the ANSYS FEM code for the evaluation of the threshold critical impact energy needed for the onset of impact induced delaminations in composite laminated structures. This approach is based on the equivalence between the stresses induced in very thin plates by a low velocity impact and by the static application of the impact load. Indeed, this equivalence is verified for very thin plates characterized by delaminations mainly caused by interlaminar shear stresses.

The kinetic energy related to an impact event can be expressed by the energy-balance equation:

$\frac{1}{2} M V^{2}=E_{c}+E_{e}$

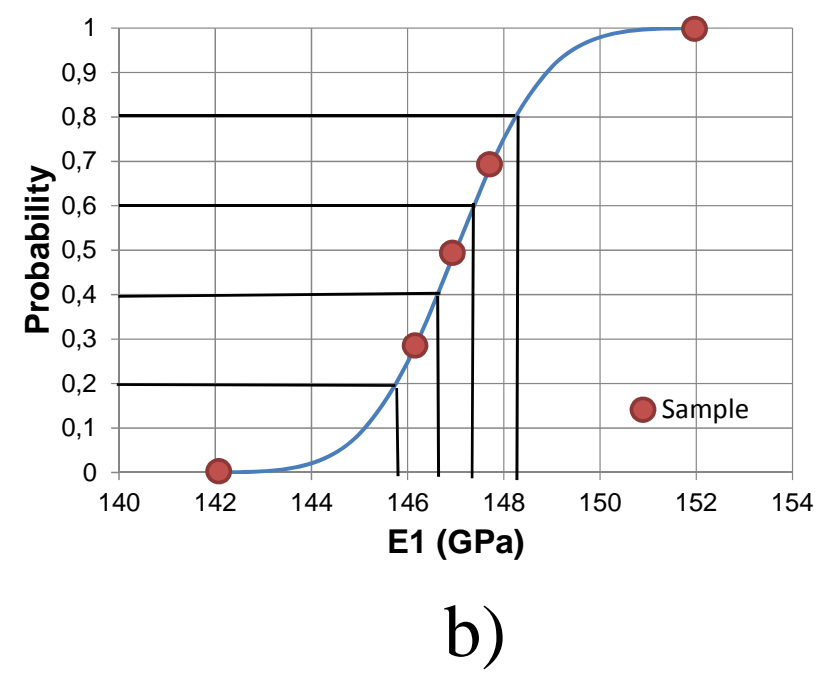

Fig. (2). Random (a) versus latin hypercube sampling method (b). 
where $M$ is the impactor mass, $V$ is the impactor velocity, $E_{e}$ is the overall energy associated to the elastic deformation of the plate during the impact event and $E_{c}$ is the energy due to the local plastic deformation caused by the indentation of the impactor on the laminate.

The Energy related to the indentation is given by the following expression [24]:

$$
E_{c}=\int_{0}^{\alpha_{m}} F d \alpha \rightarrow E_{c}=\frac{k}{n+1}\left(\frac{F_{m}}{k}\right)^{n+\frac{1}{n}}
$$

where $F=k \alpha^{n}$ represents a general accepted contact law for composite laminates, $F_{m}$ is the maximum impact force, $k$ is the contact stiffness, $\alpha$ is the indentation and $n$, in case if Hertzian contact, is a constant equal to 1.5. For carbon epoxy systems the value of $k$ is typically $130 \mathrm{MN} / \mathrm{m}^{\frac{3}{2}}$.

Assuming a linear trend for the force-displacement curve $F=F(\delta)$, the energy $E_{e}$ related to the overall elastic deformation of the composite laminate can be evaluated as follows:

$$
E_{e}=\int_{0}^{\delta_{m}} F d \delta \rightarrow E_{e}=\frac{1}{2} F d
$$

where $F$ represents the maximum impact force and $d$ the maximum displacement due to the impact.

The impact damage resistance can be measured by the threshold critical impact energy able to onset a delamination. In [12] an expression for the threshold critical impact load causing impact induced delamination onset in composite laminates subjected to low energy impacts is proposed (see equation (5)) provided that the following fundamental hypothesis are verified: circular delamination, thin plate, quasi-isotropic stacking sequence and elastic axialsymmetric deformation regime.

$$
F_{c r}=\frac{1}{3} \pi \cdot t^{\frac{3}{2}} \cdot \sqrt{8 G_{I I c} \frac{E}{1-v^{2}}}
$$

As it can be seen, the threshold load for impact induced delamination onset is a function of the mode II inter-laminar fracture toughness $G_{I I c}$, the equivalent bending stiffness and Poisson ratio of the laminate $(E$ and $v)$ and the laminate thickness $t$.

Equation (5) can be easily derived by the expression of the energy release at the boundary of a central circular delamination:

$$
G_{I I}=\frac{9 F^{2}\left(1-v^{2}\right)}{8 \pi^{2} E t^{3}}
$$

By substituting the critical energy release rate $G_{I I c}$, equation (6) is able to provide the critical impact threshold force $F_{c r}$, for instant delaminations. The absence of the delamination radius in equations (5) and (6) justifies why the increase in damage is so sudden and indeterminate at a force threshold.
Once the threshold critical impact force has been reached, multiple delaminations can arise in the thickness of the plate. Assuming that the delaminations envelope is due to exceeding the allowable inter-laminar shear stress $\tau$, it is possible, as stated in [12], to evaluate the equivalent circular delamination area for each impact force $F>F_{c r}$ from the expression:

$$
A=\frac{9}{16 \pi t^{2}}\left(\frac{F}{\tau}\right)^{2}
$$

Knowing the threshold critical impact force causing the onset of delamination, in order to find the threshold critical impact energy, according to equation (4), the maximum displacement $d_{c r}$ corresponding to the application of the impact force $F_{c r}$ is needed. This displacement can be found by performing a linear finite element static analysis simulating the impact event on the composite laminate as schematically shown in Fig. (3).

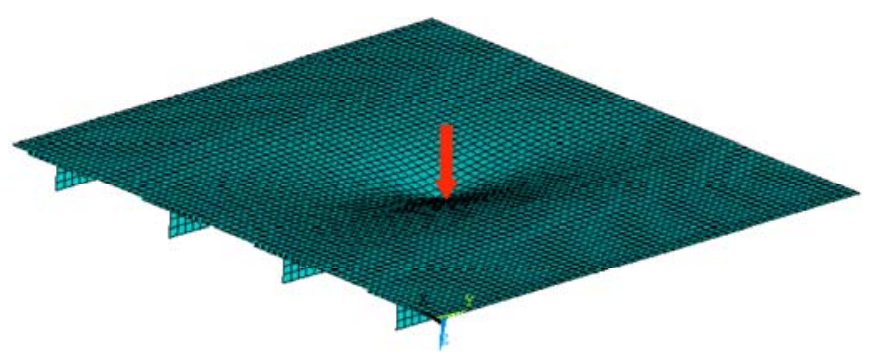

Fig. (3). FEM response to an applied impact force.

Substituting $d_{c r}$ and $F_{c r}$ in equation (4) it is possible to find the elastic part of the threshold critical impact energy as:

$E_{c r e}=\frac{1}{2} F_{c r} d_{c r}$

Finally, the total threshold critical impact energy, representative of the impact damage resistance of the composite laminate is obtained substituting the critical parameters into the equation (2):

$E_{c r}=\frac{k}{n+1}\left(\frac{F_{c r}}{k}\right)^{n+\frac{1}{n}}+\frac{1}{2} F_{c r} d_{c r}$

It is important to note that according to the hypothesis of elastic axial-symmetric deformation regime it is possible use this formula only when the contribution related to the elastic deformation exceeds the one related to the indentation ( $E_{c r e} \geq E_{c r c}$ ) and when the deformation and stress distributions are axial-symmetric around the impact location. Some considerations on the applicability of equation (5), to an analyzed stiffened panel configurations, are reported in the next sections.

According to the presented approach, if a composite laminate is impacted at a specific location with an energy greater then $E_{c r}$, then the laminate is not impact damage resistant at that location and impact induced delaminations start. 
The impact damage resistance procedure presented here involves analytical expressions and linear Finite Element analyses; hence, due to its low computational cost, it is particularly, suitable for Monte Carlo simulations.

\section{NUMERICAL APPLICATIONS: ASSESSMENT OF THE INFLUENCE OF UNCERTAINTIES ON THE IMPACT DAMAGE RESISTANCE OF COMPOSITE PANELS}

The two numerical applications introduced in this section are aimed to investigate the influence of material properties and impact location uncertainties on the impact damage resistance of composite panels. A first numerical application is presented to quantify the influence of material properties uncertainty on the impact damage resistance of a flat composite panel. A further numerical application is introduced, where the variability of the mechanical properties, due to environmental effects, and the randomness of the impact location are taken into account, simultaneously, for the determination of the impact damage resistance of a stiffened composite panel.

\subsection{Probabilistic Assessment of the Damage Resistance of a Flat Composite Panel}

The specimen introduced, in the frame of the first numerical application, is a composite flat laminate $(500 \mathrm{~mm}$ wide and $600 \mathrm{~mm}$ long). The material system considered is the carbon/epoxy T700GC-M21. The panel has a total thickness of $1 \mathrm{~mm}$ made up of $0.125 \mathrm{~mm}$ thick plies stacked according to the sequence $\left[0^{\circ} / 45^{\circ} / 45^{\circ} / 90^{\circ}\right]_{\mathrm{s}}$. The panel has been clamped on two sides and the Finite Element Model has been assembled using the 8-noded SHELL 99 layered element available in ANSYS [19] as shown in Fig. (4).

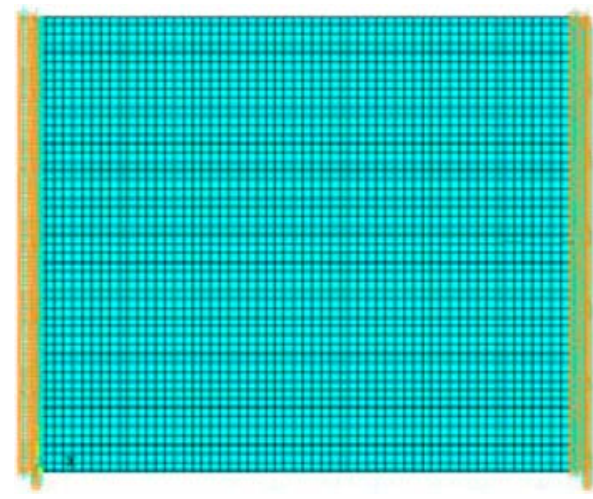

Fig. (4). Finite element model of the flat panel.

For this first numerical application, the impact location is fixed (panel centre) since the focus is the variability of material properties. The random Sampling technique is adopted for the Monte Carlo simulations which generates samples according to the corresponding statistical distribution functions of the stochastic input parameters in a random manner. The deterministic and random input parameters for the analysis have been preliminary specified. The parameters defining the geometry configuration, applied load, geometric constraints, location of the impact force and Poisson's ratio are considered as deterministic inputs so, only mean values have been specified for them. While, for the in plane elastic moduli, in plane shear modulus and for the mode II inter-laminar fracture toughness, statistical distribution functions have been defined according to data available from an experimental program on the material system M21/T700GC with temperature conditions ranging from $-55^{\circ}$ to $90^{\circ}$ and moisture percentage ranging from dry to $85 \%$. The statistical distributions of the random input material properties have been assumed to be Gaussian (normal), characterized by two parameters, namely the mean value $\mu$ and the standard deviation $\sigma$. This assumption has been suggested by the symmetrical distribution (both for temperature and moisture changes) of the material properties measurements with respect to their mean values. It is important to emphasize that, since the scope of this paper is to assess the effects of the overall environmental conditions, for each material property, the measurements at different temperature and moisture levels have been simply merged in a single probabilistic distribution. The mean and standard deviation of the M21/T700CG ply mechanical properties and their distribution function are respectively reported in Table $\mathbf{1}$ and in Fig. (5).

The number of loops chosen for the Direct Monte Carlo Simulation has been set to 1000 to ensure reliable results. Indeed the level of confidence for the probabilistic analysis has been found to be $95 \%$ for 1000 samples. In Fig. (6) the histogram plots of the input probabilistic variables are shown. These plots, obtained by dividing the whole range of variability into equal-size intervals and determining how many samples fall within each interval, provide a helpful tool to verify if the generated samples follow the specific input variables distribution functions and indicate if the number of samples (and simulations) is sufficient to perform a reliable analysis. The plots shown in Fig. (6) clearly show that the number of 1000 simulations is sufficient. Indeed for all the variables the histogram bars are close to the curves that are derived from the distribution functions (red curves), are almost "smooth" (without large "steps") and do not have recurrent major gaps.

As output of the Direct Monte Carlo simulations for this first numerical application, the cumulative distribution functions (CDF) of the threshold critical impact load (equation (5)) and of the threshold critical impact energy (equation (9)) have been investigated. Indeed, these two quantities can be considered representative of the capability of the panel to avoid impact induced delamination formation.

Table 1. Means and Standard Deviations of the Probabilistic Input Variables (Mechanical Properties of the M21/T700 ply)

\begin{tabular}{|c|c|c|c|}
\hline Property & Standard & Mean Value $(\mu)$ & Standard Deviation $(\sigma)$ \\
\hline Longitudinal tensile modulus $\left(\mathrm{E}_{1}\right)$ & EN2561 & $147000 \mathrm{MPa}$ & $1410 \mathrm{MPa}$ \\
\hline In-plane shear modulus $\left(\mathrm{G}_{12}\right)$ & EN6031 & $3840 \mathrm{MPa}$ & $1010 \mathrm{MPa}$ \\
\hline Mode II inter-laminar fracture toughness $\left(\mathrm{G}_{\mathrm{IIC}}\right)$ & EN6034 & $1447.5 \mathrm{~J} / \mathrm{m}^{2}$ & $110.8 \mathrm{~J} / \mathrm{m}^{2}$ \\
\hline
\end{tabular}




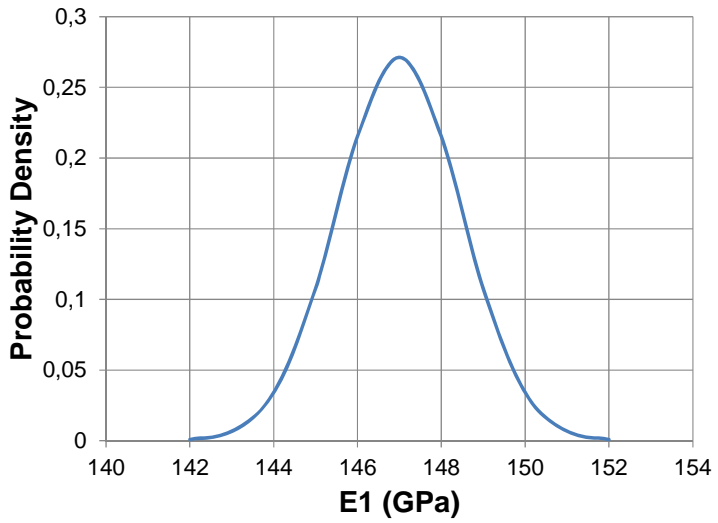

(a)

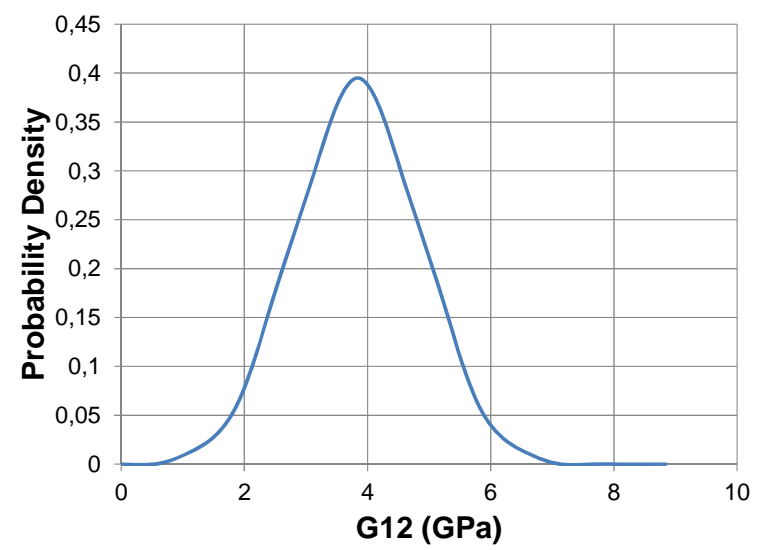

(c)

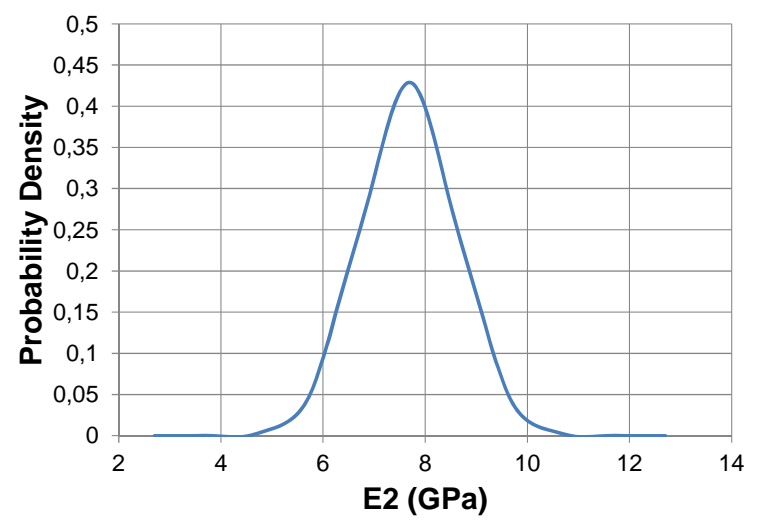

(b)

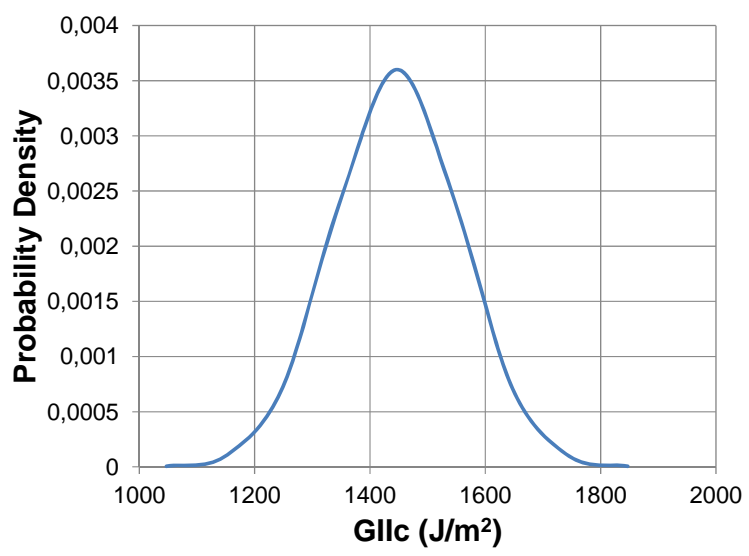

(d)

Fig. (5). Distribution function of the random input variables: (a) longitudinal elastic modulus, (b) transversal elastic modulus, (c) in-plane shear modulus, (d) mode II inter-laminar fracture toughness.

The threshold critical impact load takes into account (as shown in equation (5)) the intrinsic capability of the panel to resist to impact depending on its material and geometrical configuration while the threshold critical impact energy takes into account the influence of the elastic behavior of the panel on its capability to absorb impact energy without reaching the threshold critical impact load and generating impact induced delaminations.

As a matter of fact, these cumulative distribution functions, shown in Fig. (7), can be interpreted as reliability curves for the panel impact damage resistance.

Indeed, Fig. (7a, b) provides the relation between $F_{c r}$ $\left(\mathrm{E}_{\mathrm{cr}}\right)$, and the probability to find a specimen with a threshold critical impact force (energy) below $F_{c r}\left(E_{c r}\right)$, among the 1000 considered samples characterized by the material properties variations given by the Gaussian distributions of Fig. (5).

Looking at Fig. (7), the cumulative distribution functions show that the maximum values found for $F_{c r}$ and $E_{c r}$, in this probabilistic simulation, are, respectively, $1280 \mathrm{~N}$ and 350

$J$. This means there is certainty ( $100 \%$ probability) to find samples with lower critical force and energy. On the other hand, the minimum values found for $\mathrm{F}_{\mathrm{cr}}$ and $\mathrm{E}_{\mathrm{cr}}$, in this probabilistic simulation are, respectively, $980 \mathrm{~N}$ and $219 \mathrm{~J}$. Indeed, the probability to find sample with lower $F_{c r}$ and $E_{c r}$ is $0 \%$. Hence these minimum values can be considered as the most conservative threshold critical impact force and energy for the analyzed panel resulting from the performed probabilistic analysis.

It is important to underline that a standard deterministic analysis, considering only the mean values of the distributions reported in Fig. (5), is able to return only a single value for the threshold critical force and energy (respectively $1129.2 \mathrm{~N}$ and $286.8 \mathrm{~J}$ ), which is not able to take into account the properties variations due to environmental conditions and the probability that the panel truly resists to a specific impact energy without developing impact induced delaminations.

Stochastic simulations give an additional fundamental information if compared to the deterministic ones: the sensitivity analysis results highlighting the input parameters that mostly influence the damage resistance properties. The sensitivities results for $\mathrm{F}_{\mathrm{cr}}$ and $\mathrm{E}_{\mathrm{cr}}$, are presented, respectively in Fig. (8a, b). 


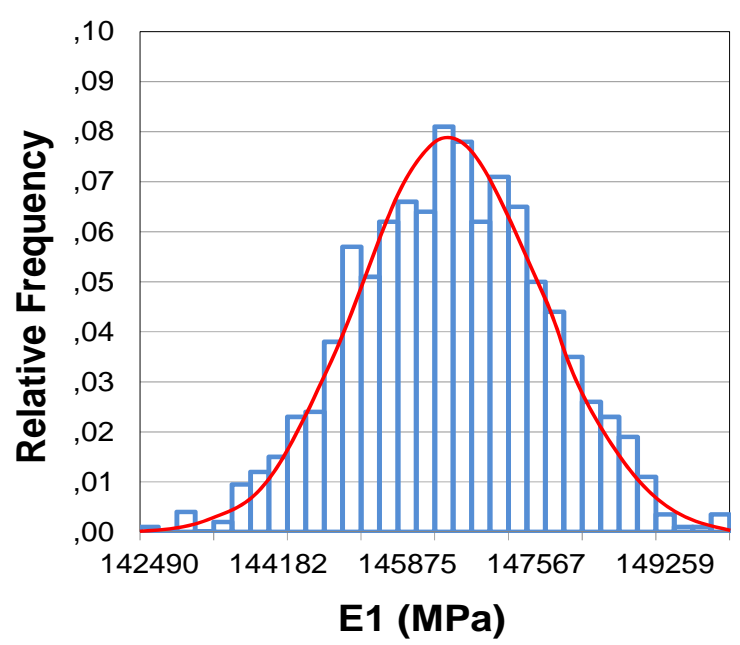

(a)

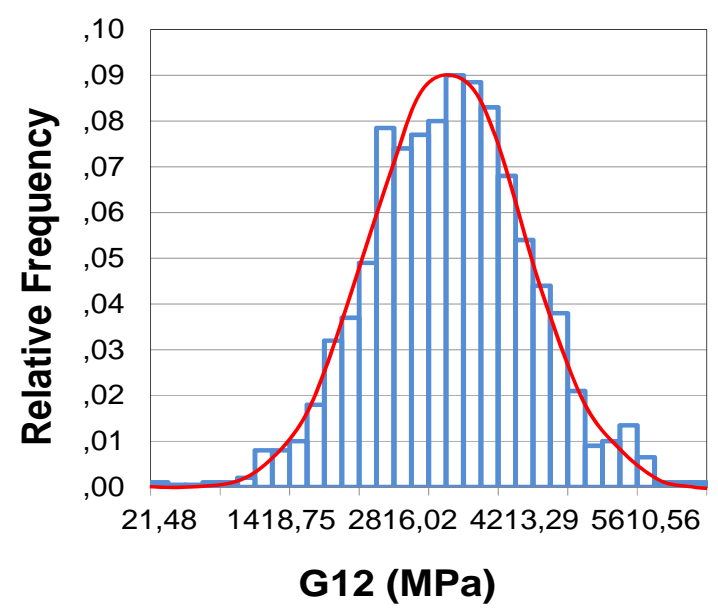

(c)

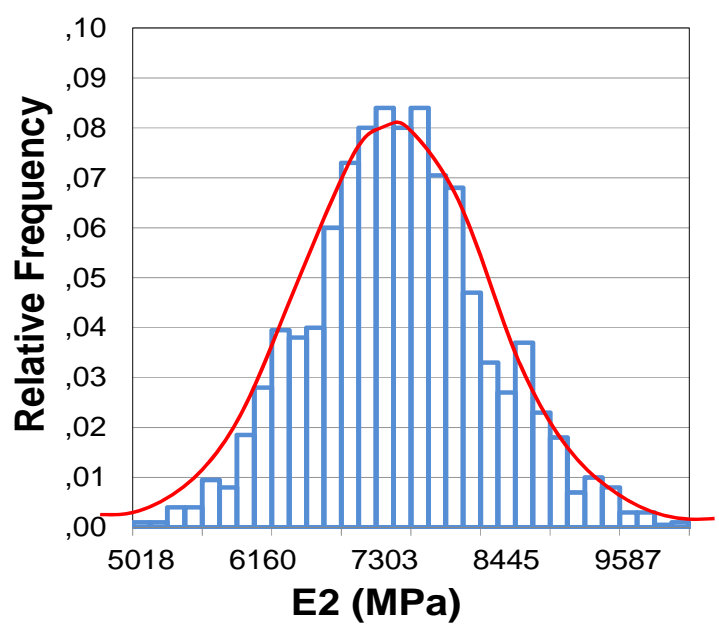

(b)

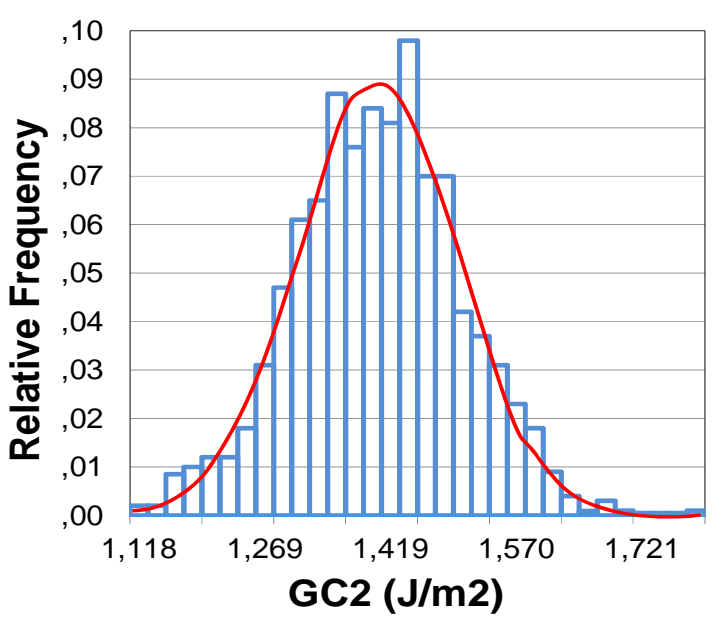

(d)

Fig. (6). Assigned material properties (histograms) V.s. input Distribution function of the random input variables (line): (a) longitudinal elastic modulus, (b) transversal elastic modulus, (c) in-plane shear modulus, (d) mode II inter-laminar fracture toughness.

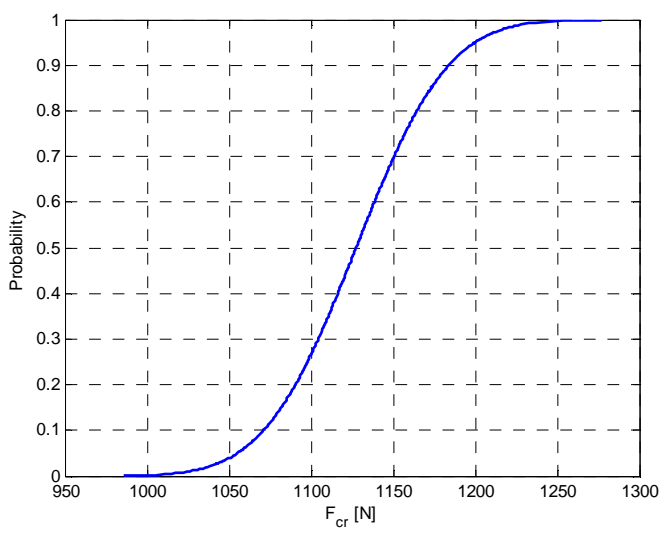

(a)

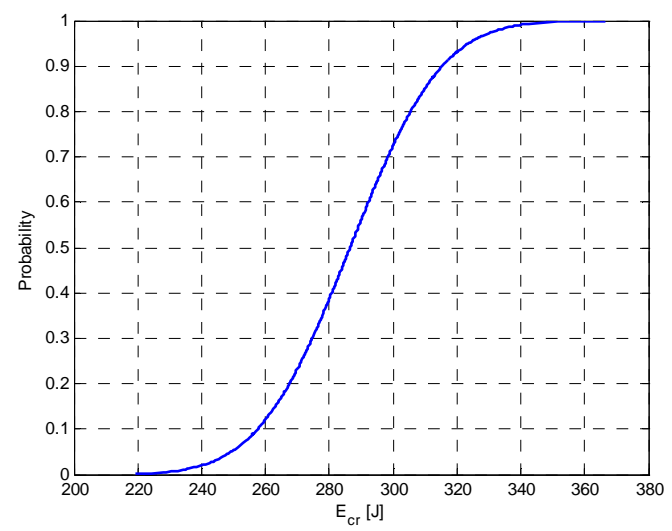

(b)

Fig. (7). $F_{c r}(a)$ and $E_{c r}(b)$ cumulative distribution function. 
Output parameter Fcr (Linear correlation sensitivity results) - plane panel

a)

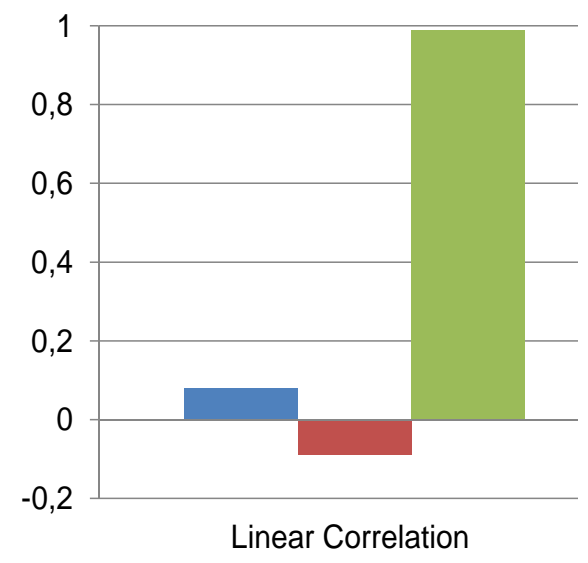

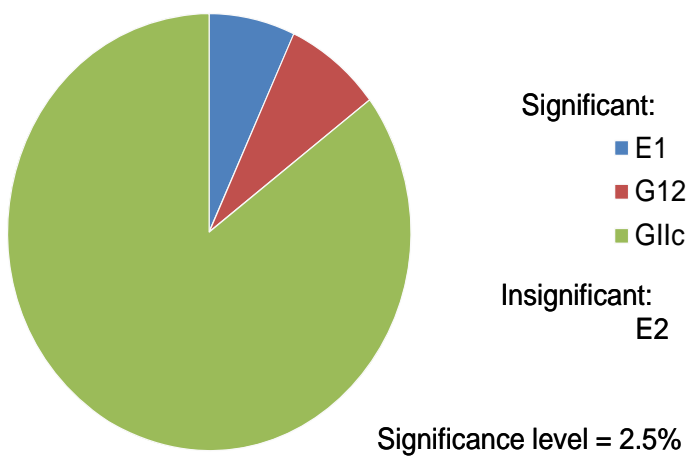

Output parameter Ecr (Linear correlation sensitivity results) - plane panel

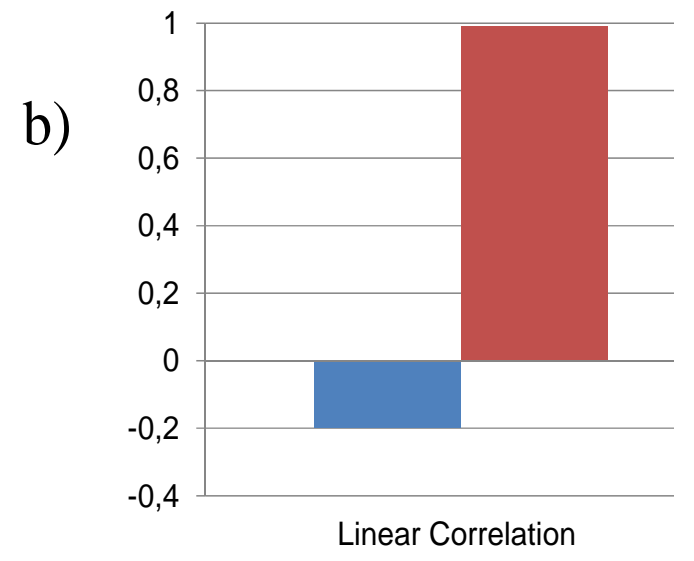

Fig. (8). $F_{c r}\left(\right.$ a) and $E_{c r}(b)$ linear correlation sensitivity results.

The Pearson linear correlation coefficient has been used to evaluate the sensitivities of the probabilistic input variables. This coefficient is defined according to the following expression:

$$
r_{p}=\frac{\sum_{i=1}^{n}\left(x_{i}-\bar{x}\right)\left(y_{i}-\bar{y}\right)}{\sqrt{\sum_{i=1}^{n}\left(x_{i}-\bar{x}\right)^{2}} \sqrt{\sum_{i=1}^{n}\left(y_{i}-\bar{y}\right)^{2}}}
$$

Being $X$ and $Y$ probabilistic variables of size n number of simulation loops) represented as $\left[x_{1}, x_{2}, \ldots, x_{n}\right]^{T}$ and $\left[y_{1}, y_{2}, \ldots, y_{n}\right]^{T}$ with means defined respectively as $\bar{x}=\frac{1}{n} \sum_{i=1}^{n} x_{i}$ and $\bar{y}=\frac{1}{n} \sum_{i=1}^{n} y_{i}$. The correlation coefficient $r_{p}$ usually yields a small, but nonzero value, it ranges

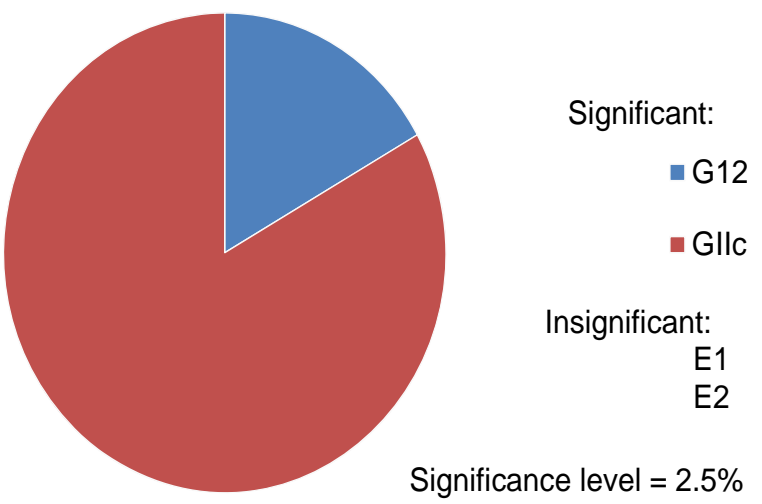

between 1 and -1, approaching these limits correspond to the maximum correlation between $X$ and $Y$. If $r_{p}=0$ then $X$ and $Y$ are not correlated.

In Fig. (8), sensitivities are visualized both as a bar chart and a pie chart. In the bar chart the most important random input variable, with the highest sensitivity, appears in the rightmost position and the others follow to the left in the order of their importance. A positive sensitivity indicates that increasing the value of the random input variable increases the value of the random output parameter too. Likewise, a negative sensitivity indicates that increasing the random input variable, the random output parameter is reduced. In the pie chart, sensitivities are relative to each other. In a pie chart the random input variable with the highest sensitivity will appear first at the 12 o'clock position, and the others follow in anticlockwise direction in the order 
of their importance. Probabilistic input variables with significance under $2.5 \%$ are not shown.

From Fig. (8), it is possible to notice that the interlaminar mode II fracture toughness $G_{\text {IIc }}$ is the input parameter whose variations mostly influence both the threshold critical impact force and energy. On the other hand, the influence of the shear stiffness modulus $G_{12}$ variations on the threshold values is significantly less appreciable. The longitudinal stiffness modulus $E_{l}$ variations seem to have a marginal influence on the threshold critical impact force while they are insignificant (significance under $2.5 \%$ ) for the threshold critical impact energy. The transversal stiffness modulus $E_{2}$ variations are insignificant for both the threshold quantities.

\subsection{Probabilistic Assessment of the Damage Resistance of a Stiffened Panel}

The specimen considered, for the second numerical application, is a stiffened composite with variable mechanical properties, due to environmental effects, and random location of the impact force.

The analysed stiffened panel is $500 \mathrm{~mm}$ wide and 600 $\mathrm{mm}$ long with the skin made of 24 plies and the stringers made of 10 plies (the material system is again the carbon/epoxy T700GC-M21). Details on the geometric configuration of the panel are given in Fig. (9) while the staking sequence of the skin and of the stiffeners components are shown in Table 2.

Table 2. Stacking Sequence of the Stiffened Panel SubComponents

\begin{tabular}{|c|c|}
\hline Panel Sub-Component & Stacking Sequence \\
\hline \hline Skin & {$[-45,0,45,90,-45,0,45,90,-45,0,45,90]_{\mathrm{S}}$} \\
\hline Stringer base & {$[45,-45,0,0,90]_{\mathrm{s}}$} \\
\hline Stringer web & {$[45,-45,0,0,90,90,0,0,-45,45]_{\mathrm{S}}$} \\
\hline
\end{tabular}

The finite element model is introduced in Fig. (10).

The stiffened panel has been clamped on the two transversal edges with blocked rotations on the other two edges. The Finite Element Model has been built again using the 8-noded SHELL 99 layered element available in ANSYS [19].

As remarked, to evaluate the damage resistance, in addition to the variability of the mechanical properties of the material, the randomness of the position of the force on all over the panel is taken into account. Since the probability of

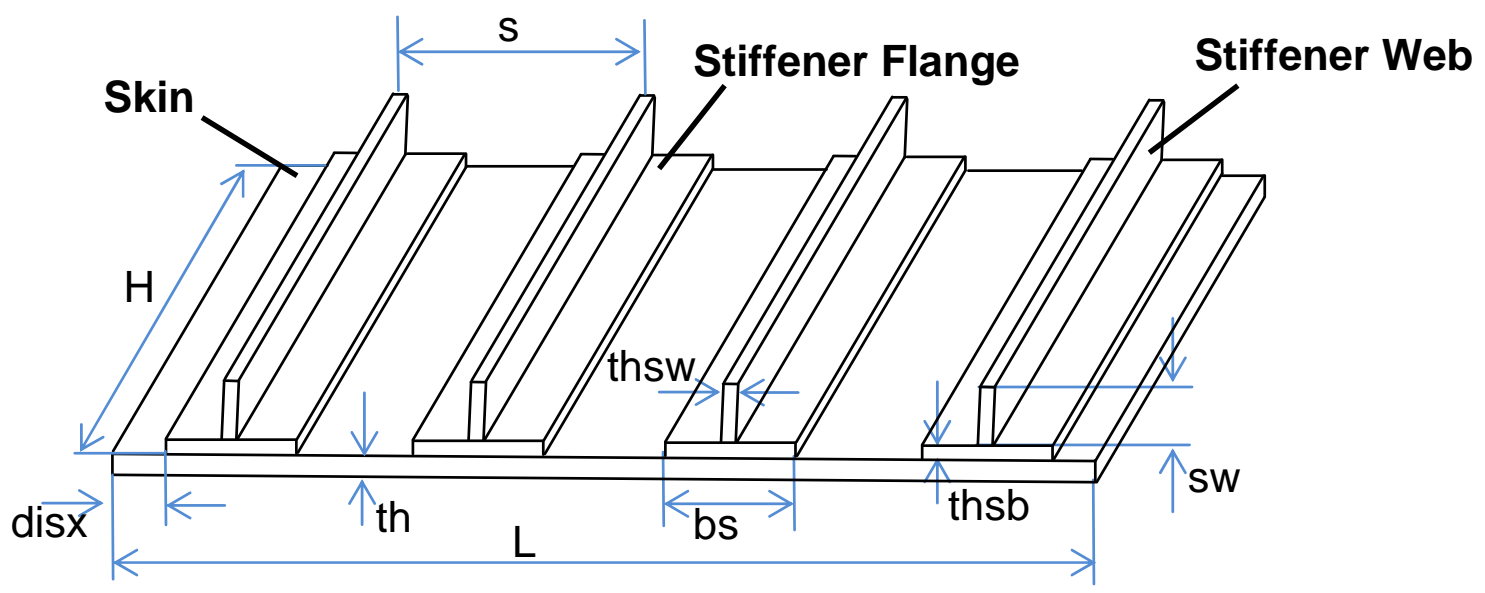

\begin{tabular}{|c|c|}
\hline$L$ & $600 \mathrm{~mm}$ \\
\hline$H$ & $500 \mathrm{~mm}$ \\
\hline$b s$ & $52,27 \mathrm{~mm}$ \\
\hline$d i s x$ & $20,365 \mathrm{~mm}$ \\
\hline$t h$ & $4,512 \mathrm{~mm}$ \\
\hline$t h s b$ & $1,88 \mathrm{~mm}$ \\
\hline$t h s w$ & $3,76 \mathrm{~mm}$ \\
\hline$s$ & $169 \mathrm{~mm}$ \\
\hline$s w$ & $26,14 \mathrm{~mm}$ \\
\hline
\end{tabular}

Fig. (9). Geometrical configuration of the stiffened panel. 
impact is uniformly distributed all over the stiffened panel, the coordinates of the impact location $\left(x_{F}, y_{F}\right)$ can be treated as random input variables with the uniform distribution functions plotted in Fig. (11).

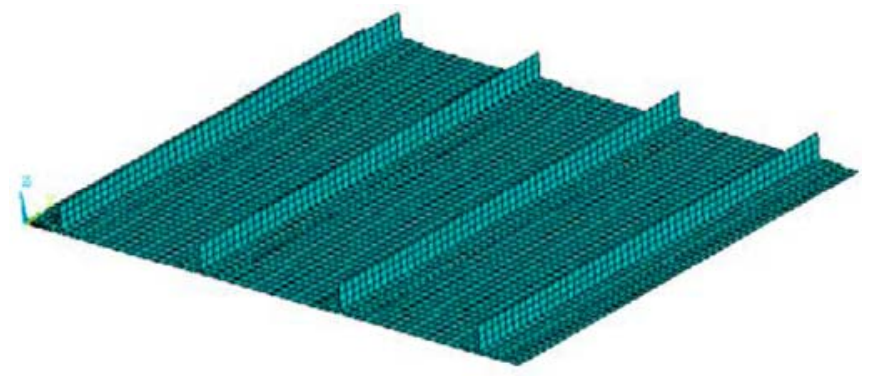

Fig. (10). Stiffened panel finite element model.

Concerning the random material properties, the distributions of Fig. (5) have been used. For this numerical application, both the random impact location coordinates $\left(x_{F}, y_{F}\right)$ and the random material properties are chosen from the assigned distribution functions using the Latin Hypercube Monte Carlo's sampling method. The number of loops chosen for this Monte Carlo Simulation has be set to 600 to ensure reliable results. Indeed the level of confidence for the probabilistic analysis has been found to be $95 \%$ for 600 samples without repetitions thanks to the Latin Hypercube sampling method. Moreover, the histogram plots of the input probabilistic variables, shown in Fig. (12), clearly show that the number of 600 simulations is sufficient, being, for all the variables, the histogram bars very close to the curves that are derived from the distribution functions of the probabilistic variables given in input.

For the stiffened composite panel, only the cumulative distribution function of the threshold critical impact energy has been considered as a relevant output of the Monte Carlo simulations. As for the previous numerical application, the threshold critical impact energy takes into account the influence of the elastic behavior of the stiffened panel on its capability to absorb impact energy without inducing delaminations.

Actually, the threshold critical impact force has not been considered as a relevant output since it is affected by a strong step variation as the impact location moves from bays to stringer feet (mainly due to the difference in thickness of

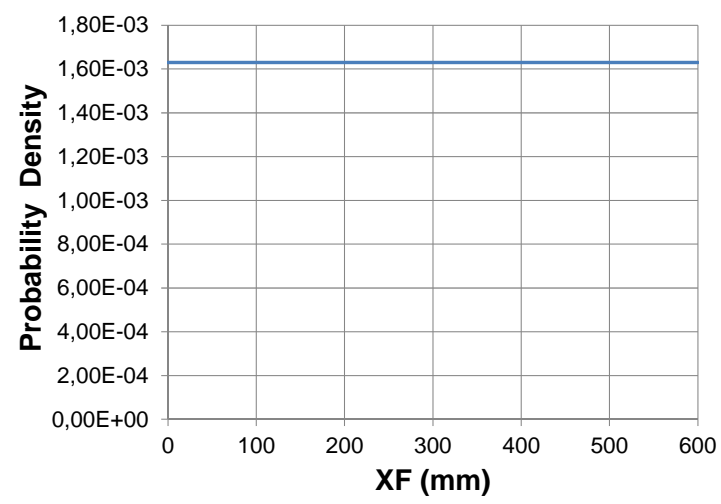

(a) the panel at bay and at stringer feet locations). This step variation is considerably larger than the variations due to material properties and impact location uncertainties as shown in Fig. (13).

The Cumulative Distribution Function of the threshold critical energy, shown in Fig. (14), can be interpreted as a reliability curve for the stiffened panel impact damage resistance.

Looking at Fig. (14), the cumulative distribution function shows that the maximum value found for $E_{c r}$, in this probabilistic simulation, is $75 \mathrm{~J}$. This means there is certainty (100\% probability) to find samples with lower critical energy. On the other hand, the minimum values found for $E_{c r}$, in this probabilistic simulation, is $9 J$. Indeed, the probability to find a sample with lower $E_{c r}$ is $8 \%$. As a matter of fact, in the frame of this application, 48 samples ( $8 \%$ of the total number of samples), characterized by an impact location close to the boundaries of the panel and close to the center line of the stringers, have not been considered reliable. Indeed at the boundaries of the panel and at the center line of the stringers, the proposed impact damage resistance model, described by equations (5),(6) and (7), is clearly not applicable due to the lack of axialsymmetry in deformations and stress distributions.

As a consequence, these 48 samples have not been included in the cumulative distribution representation. Indeed a preliminary study on the applicability of equations (5),(6) and (7) to stiffened panel configurations, characterized by a thicker skin and thinner stringer feet, as the ones introduced in this paper, has been performed. This study has outlined that small portions of the panels, located at boundaries and close to the center line of stringers, subjected an impact threat develop significant non-axialsymmetric deformation and stress fields due respectively to non-axial-symmetric boundary conditions and non-axialsymmetric geometry. As parameter to check the applicability of equations (5),(6) and (7), the ratio $R$ between non axialsymmetric and axial-symmetric averaged stresses has been considered. Considering the damage resistance criterion applicable to the impact locations fulfilling the requirement of $R<1 / 3$, about the $10 \%$ of the panel surface has been excluded from the damage resistance computations. It is important to underline that, for the full applicability of

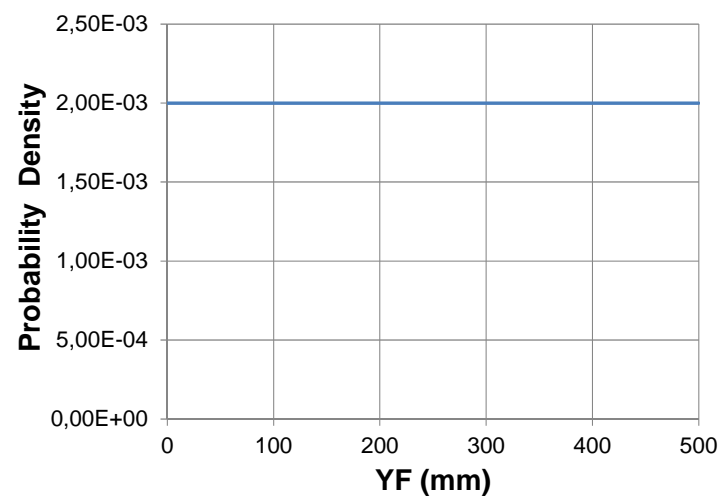

(b)

Fig. (11). Uniform distribution function of the impact location coordinates. 


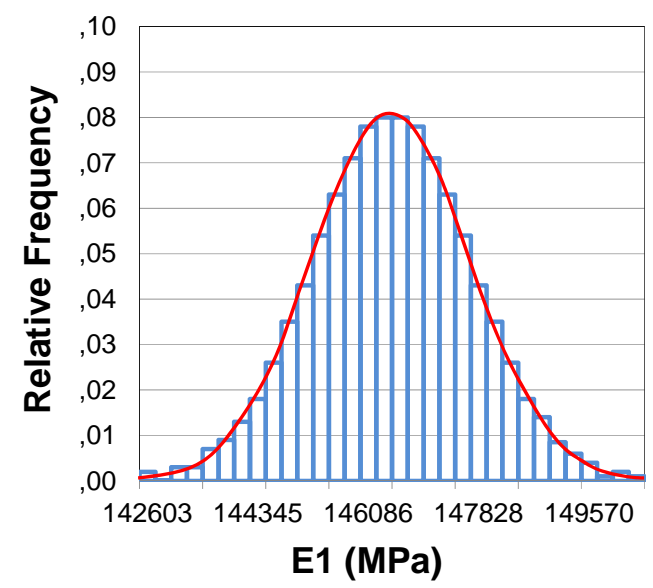

(a)

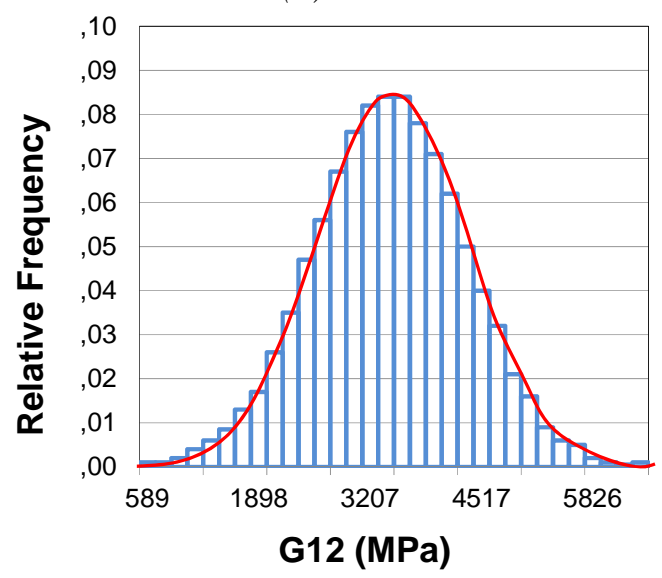

(c)

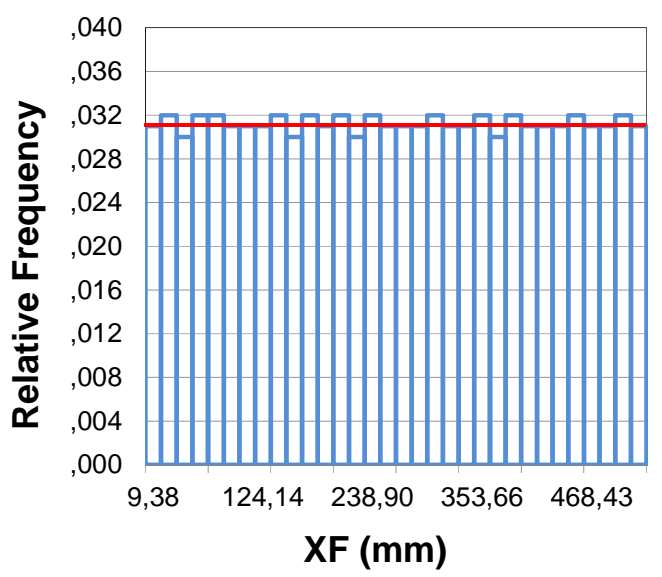

(e)

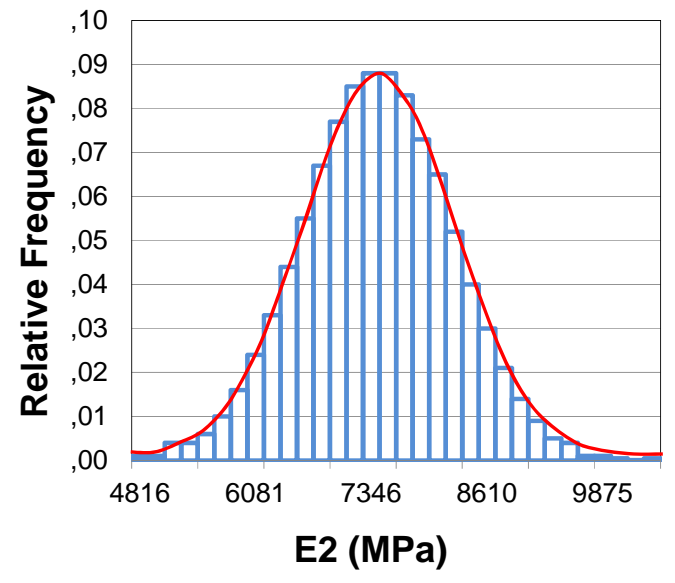

(b)

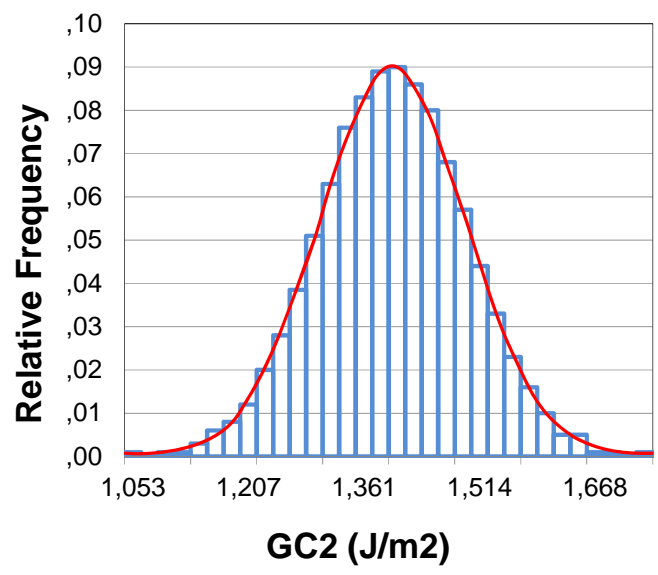

(d)

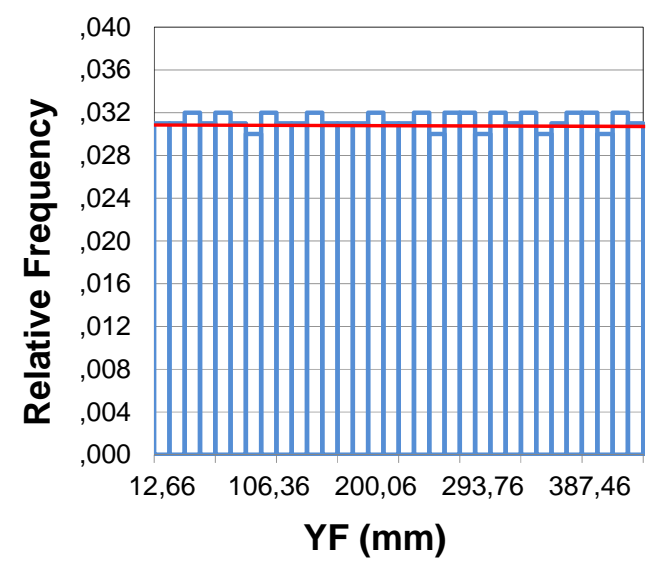

(f)

Fig. (12). Assigned (histograms) V.s. input Distribution function of the random input variables (line): (a) longitudinal elastic modulus, (b) transversal elastic modulus, (c) in-plane shear modulus, (d) mode II inter-laminar fracture toughness, (e) impact location $\mathrm{x}_{\mathrm{F}}$ coordinate, (f) impact location $\mathrm{y}_{\mathrm{F}}$ coordinate.

equations (5),(6) and (7), only bay impacts far from the boundaries of the panel should have been considered. However, for the purposes of this paper, which is intended to provide information demonstrating the usefulness of a probabilistic approach for the evaluation of the damage resistance of complex composite structures, the level of accuracy reached, excluding from computations only this small portions of the panel surface, can be still considered acceptable. 


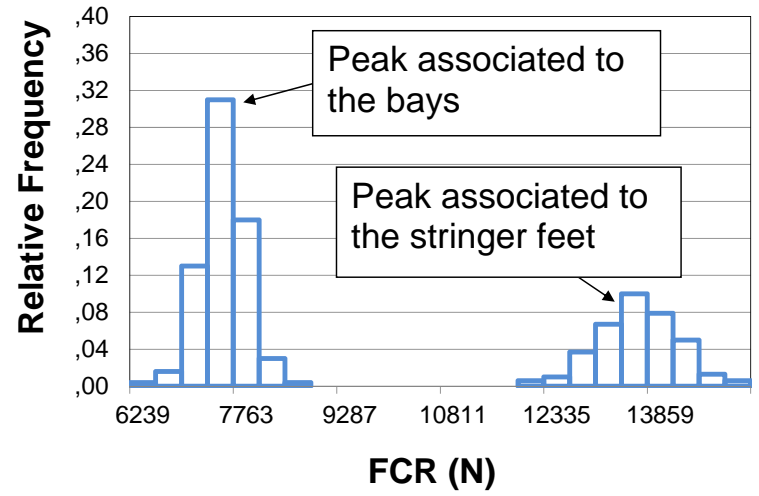

Fig. (13). $F_{c r}$ distribution function for the stiffened panel.

As seen for the previous numerical application, the minimum value $(9 J)$ can be considered as the most conservative threshold critical impact energy for the analyzed stiffened panel resulting from the performed probabilistic analysis.

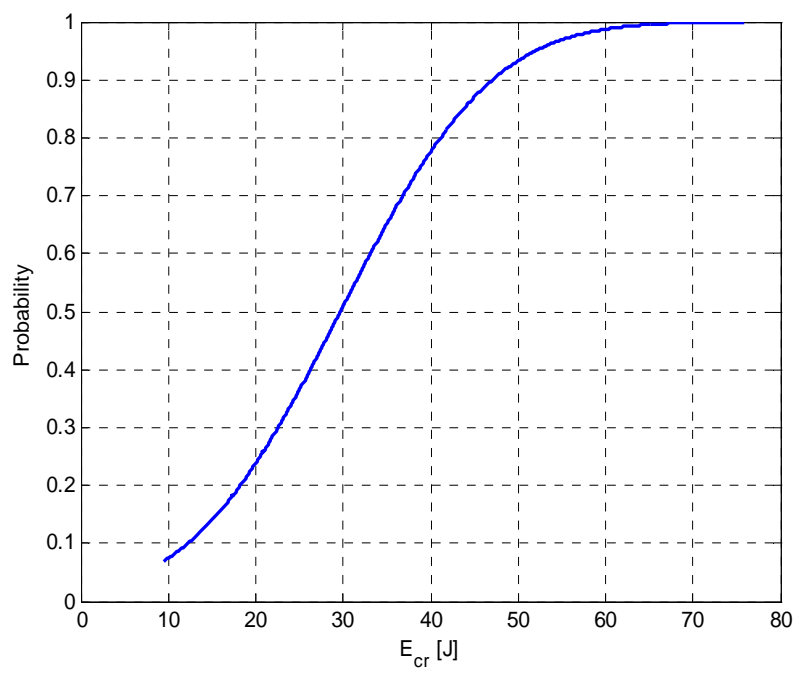

Fig. (14). $E_{c r}$ cumulative distribution function for the stiffened panel.

In order to compare our probabilistic results with deterministic results, as done for the flat panel application, some considerations need to be pointed out concerning the way to take into account the presence of multiple impact locations when evaluating the impact damage resistance of a composite structure in the frame of a deterministic analysis.

Labeling with $N_{\text {stringer }}$ and $N_{\text {bay }}$ the number of impact locations considered on stringer feet and bays, it is possible to evaluate the mean values of the threshold critical impact energy associated to the bays and to the stringer feet, starting from the deterministic threshold critical impact energy associated to each impact location, by adopting, respectively, equations (11) and (12).

$$
\hat{E}_{b a y}=\frac{\sum_{i=1}^{N_{b a y}} E_{i r}}{N_{b a y}}
$$

$$
\hat{E}_{\text {otringer }}=\frac{\sum_{i=1}^{N_{\text {stringery }}} E_{\dot{a} r}}{N_{\text {stringer }}}
$$

These mean values, in order to obtain a representative overall measure of the damage resistance of the stiffened panel considering different impact locations, should be weighted with respect to the area of the stringer feet $A_{\text {stringer }}$ and bays $A_{b a y}$ according to the following equation:

$$
\hat{E}_{c r}=\frac{\hat{E}_{\text {bay }} \cdot A_{b a y}+\hat{E}_{\text {stringer }} \cdot A_{\text {stringer }}}{A_{\text {stringer }}+A_{\text {bay }}}
$$

The threshold critical impact energy given by equation (13) has been used in this paper as deterministic measure of the impact damage resistance of stiffened composite panels.

For the stiffened panel under consideration, using, as usual, the mean values of the material properties, $\hat{E}_{c r}$ is 25.29J. Comparing this deterministic value to the probabilistic results shown in Fig. (14), it is possible to note as the deterministic analysis, once again, provides a value of the threshold critical energy, without indications on the probability to find samples with lower $E_{c r}$, being not able to take into account the properties variations due to environmental conditions and the randomness of the impact location.

The Probabilistic simulation gives, once again, additional information concerning the influence of the input parameters on the damage resistance properties. Indeed for this numerical application, using once again the Pearson linear correlation coefficient, the inter-laminar mode II fracture toughness $G_{I I c}$ and the longitudinal stiffness modulus have been found the only input parameters whose variations significantly (significance over $2.5 \%$ ) influence the threshold critical impact energy. In Fig. (15) the sensitivity results obtained for the stiffened panel are introduced.

\section{PROBABILISTIC IMPACT DAMAGE RESIS- TANCE DESIGN: COMPARISON BETWEEN TWO STIFFENED PANEL CONFIGURATIONS}

In this section, the last numerical application, aimed to compare the stochastically assessed impact damage resistance behavior of two stiffened composite panels, is introduced. The stiffened composite panels considered in the frame of this numerical application are characterized by the same weight and the same buckling load. The geometrical definition, with reference to the schematic representation of the panel given in Fig. (9), and the material stacking sequences of these two configurations are summarized in Table 3 .

The material system, the boundary conditions and the FEM model are kept unchanged with respect to the previous numerical application. The two analyzed panel configurations are shown in Fig. (16).

The deterministic values of the damage resistance for panel\#1 and panel\#2, calculated according to equation (13) and considering as input material properties the mean values of the stochastic distributions of Fig. (5), are respectively $26.15 J$ and $31.19 J$. Hence, deterministically, panel\#2 


\section{Output parameter Ecr (Linear correlation sensitivity results) - stiffened panel}
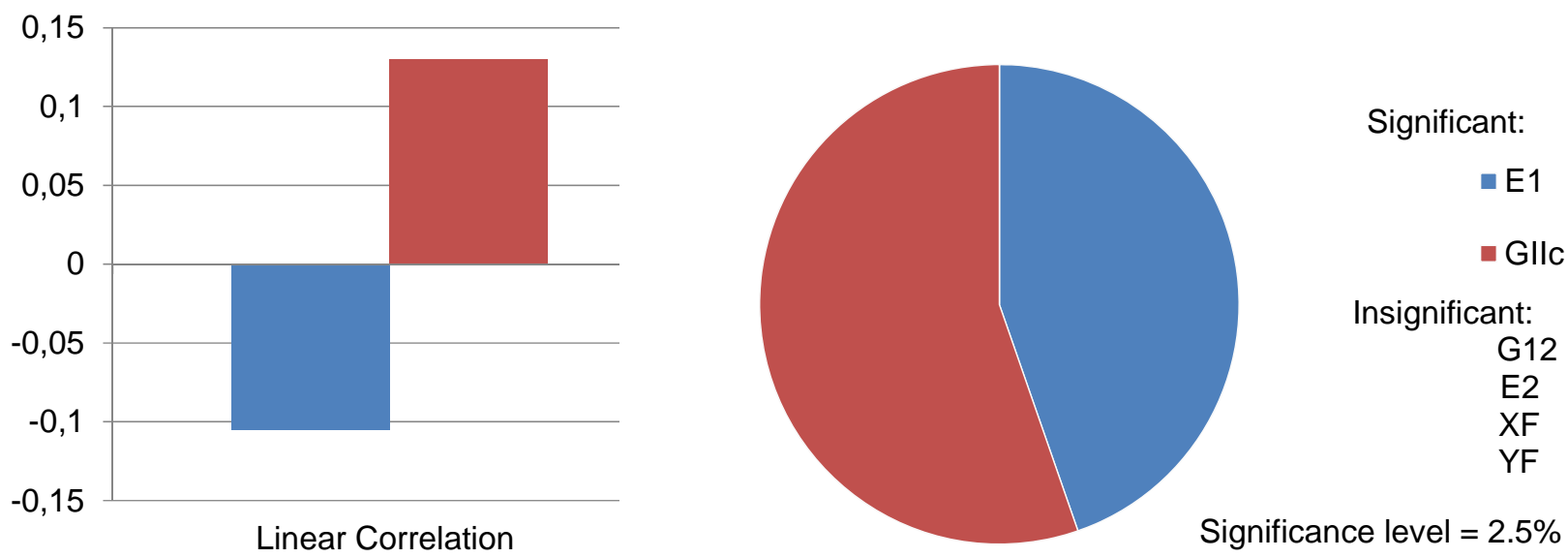

Fig. (15). $\mathrm{E}_{\mathrm{cr}}$ linear correlation sensitivity results for the stiffened panel.

exhibits the best performance in terms of impact damage resistance. This result was expected since the deterministic threshold critical impact energy of the entire panel is evaluated (see equation (13)) as an average of the mean threshold critical impact energies in the bays and in the stringers weighted by the stringers and bays areas. As a matter of fact, according to equation (5), the threshold critical impact force - and, as a consequence, the threshold critical impact energy - for impacts on stringers is higher than the one for impacts on bays due to the increased (skin + stringer foot) panel thickness in the stringer region. Hence, for panel\#2 which, differently from panel\#1, is characterized by very wide stringers feet and very narrow bays, a higher value of the overall deterministic threshold critical impact energy can be expected.

Table 3. Geometrical and Layup Parameters of the Two Stiffened Panels

\begin{tabular}{|c|c|c|}
\hline Parameter & Panel \# 1 & Panel \# 2 \\
\hline \hline $\mathrm{L}$ & $600 \mathrm{~mm}$ & $600 \mathrm{~mm}$ \\
\hline $\mathrm{H}$ & $527 \mathrm{~mm}$ & $540 \mathrm{~mm}$ \\
\hline $\mathrm{bs}$ & $47 \mathrm{~mm}$ & $82.27 \mathrm{~mm}$ \\
\hline disx & $20.365 \mathrm{~mm}$ & $20.365 \mathrm{~mm}$ \\
\hline th & $4.512 \mathrm{~mm}$ & $3.76 \mathrm{~mm}$ \\
\hline thsb & $1.5 \mathrm{~mm}$ & $1.88 \mathrm{~mm}$ \\
\hline thsw & $3 . \mathrm{mm}$ & $3.76 \mathrm{~mm}$ \\
\hline $\mathrm{s}$ & $170.8 \mathrm{~mm}$ & $159 \mathrm{~mm}$ \\
\hline sw & $26.14 \mathrm{~mm}$ & $26.14 \mathrm{~mm}$ \\
\hline $\begin{array}{c}\text { Skin stacking } \\
\text { sequence }\end{array}$ & $\begin{array}{c}-45,0,45,90, \\
-45,0,45,90]_{\mathrm{s}}\end{array}$ & $\begin{array}{c}{[45,0,90,-45,45,} \\
0,90,-45,90,0]_{\mathrm{s}}\end{array}$ \\
\hline $\begin{array}{c}\text { Stringer foot } \\
\text { stacking sequence }\end{array}$ & {$[45,-45,0,90]_{\mathrm{s}}$} & {$[45,-45,0,0,90]_{\mathrm{s}}$} \\
\hline $\begin{array}{c}\text { Stringer web } \\
\text { stacking sequence }\end{array}$ & $\begin{array}{c}{[45,-45,0,90,} \\
90,0,-45,45]_{\mathrm{s}}\end{array}$ & $\begin{array}{c}{[45,-45,0,0,90,} \\
90,0,0,-45,45]_{\mathrm{s}}\end{array}$ \\
\hline
\end{tabular}

For the probabilistic analysis, the random material properties, and the impact locations coordinates distributions, respectively, shown in Figs. $(5,11)$ have been used. Also for this numerical application, both the impact location coordinates $\left(x_{F}, y_{F}\right)$ and the random material properties have been chosen from the assigned distribution functions using the Latin Hypercube Monte Carlo's sampling method to select the 600 samples ensuring reliable results.

The $E_{c r}$ cumulative distribution functions found for the two stiffened panel configurations are compared in Fig. (17).

Indeed a criterion to compare the probabilistic performances in terms of the threshold critical impact energy could be introduced based on the comparison between the CDF curves: the curve showing the highest probability of occurrence of higher threshold critical impact energy values can be considered representative of better performance in terms of impact damage resistance. The compared CDF curves can cross each other. This event occurs when the set of threshold critical impact energies of one curve is included in the set of threshold critical impact energies of the other curve.

According to the above defined criterion, Fig. (17) shows, differently from the deterministic approach, that, from a probabilistic point of view, the panel\#1 has the best performance in terms of impact damage resistance.

As a matter of fact, this application demonstrates that the variability of the material properties and impact location can strongly influence the impact damage resistance of composite laminated structures. As a consequence the real performance in terms of damage resistance can be completely different from the one predicted by a standard deterministic approach.

\section{CONCLUSIONS}

In this paper a probabilistic procedure for the design of impact damage resistant composite laminated structures has been proposed. The probabilistic procedure, implemented in 


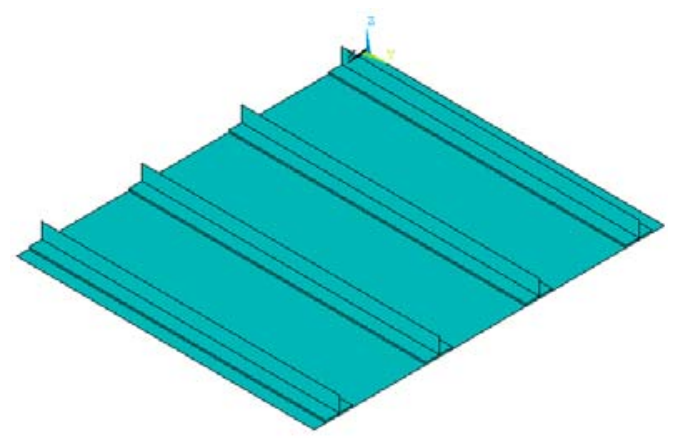

Panel configuration \#1

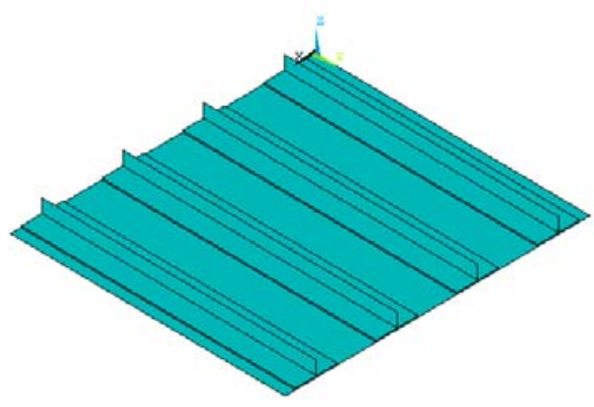

Panel configuration \#2

Fig. (16). Stiffened panel configurations compared in terms of probabilistic impact damage resistance.

the ANSYS FEM code, uses Monte Carlo Simulations to take into account the uncertainties in material properties due to changes in environmental conditions and the randomness of the impact location. The proposed numerical procedure also uses an approach based on the threshold critical impact force for the determination of the impact damage resistance.

The proposed probabilistic approach has been used to determine which are the input parameters that mostly influence the impact damage resistance properties. In general, the mode II inter-laminar fracture toughness $G_{I I c}$ has been found the most critical input variable influencing the impact damage resistance of flat and stiffened composite panels.

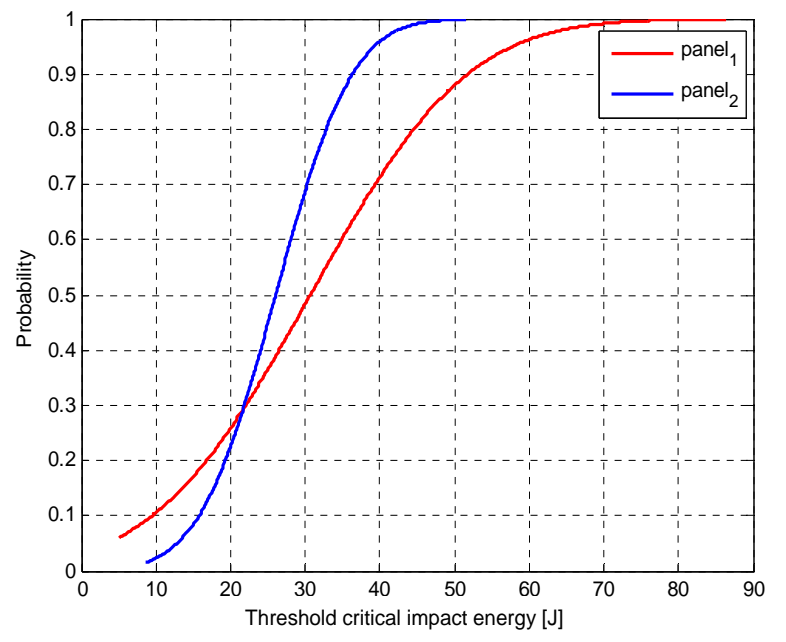

Figure 17: CDFs of the stiffened panels.

Fig. (17). CDFs of the stiffened panels.

The cumulative distribution function of the threshold critical impact energy, taking into account the elastic structural behaviour during the impact event, has been found to be the most representative output from the probabilistic analysis for the assessment of the impact damage resistance both for flat and stiffened composite panels. This cumulative distribution function relates the probability of occurrence to each threshold critical impact energy value associated to material properties and impact location variations, providing a more reliable measure of the impact damage resistance with respect to standard deterministic approaches. Finally, a design criterion has been suggested for quantifying the performance of composite panels in terms of stochastically assessed impact damage resistance. The application of this criterion has demonstrated that the variability of the material properties due to environmental conditions and the randomness of the impact location can strongly influence the impact damage resistance of composite laminated structures leading, in some cases, to a completely different performance with respect to the one predicted by a standard deterministic approach. Hence, the proposed probabilistic approach, taking into account these uncertainties, can be considered as the most suitable tool for the determination of a reliable measure of the impact damage resistance when designing composite laminated structures.

\section{CONFLICT OF INTEREST}

The authors confirm that this article content has no conflict of interest.

\section{ACKNOWLEDGEMENTS}

Declared none.

\section{REFERENCES}

[1] Evci C, Gülgeç M. An experimental investigation on the impact response of composite materials. Int J Impact Eng 2012; 43: 40-51.

[2] Cantwell WJ, Morton J. The impact resistance of composite materials - a review. Composites 1991; 22: 347-62.

[3] Hosseinzadeh R, Shokrieh MM, Lessard L. Damage behavior of fiber reinforced composite plates subjected to drop weight impacts. Compos Sci Technol 2006; 66: 61-8.

[4] Quaresimin M, Ricotta M, Martello L, Mian S. Energy absorption in composite laminates under impact loading. Compos Part B Eng 2013; 44: 133-40.

[5] Schoeppner GA, Abrate S. Delamination threshold loads for low velocity impact on composite laminates. Compos Part A Appl Sci Manuf 2000; 31: 903-15.

[6] Takeda S, Minakuchi S, Okabe Y, Takeda N. Delamination monitoring of laminated composites subjected to low-velocity impact using small-diameter FBG sensors. Compos Part A Appl Sci Manuf 2005; 36: 903-8.

[7] Park H, Kong C, Lee K. Investigation on damage tolerance of carbon/epoxy laminate for aircraft structural design. Key Eng Mat 2012; 488-489: 460-3.

[8] Corvino M, Iuspa L, Riccio A, Scaramuzzino F. Weight and cost oriented multi-objective optimisation of impact damage resistant stiffened composite panels. Compos Struct 2009; 87: 1033-42.

[9] Gigliotti M, Riccio A, Iuspa L, Scaramuzzino F, Mormile L. Weight optimisation of damage resistant composite panels with a posteriori cost evaluation. Compos Struct 2009; 88: 312-22.

[10] Davies GAO, Hitchings D, Wang J. Prediction of threshold impact energy for onset of delamination in quasi-isotropic carbon/epoxy composite laminates under low-velocity impact. Compos Sci Technol 2000; 60: 1-7. 
[11] Schoeppner GA, Abrate S. Delamination threshold loads for low velocity impact on composite laminates. Compos Part A Appl Sci Manuf 2000; 31: 903-15.

[12] Davies GAO, Zhang X. Impact damage prediction in carbon composite structures. Int J Impact Eng 1995; 16: 149-70.

[13] Botelho EC, Pardini LC, Rezende MC. Hygrothermal effects on the shear properties of carbon fiber/epoxy composites. J Mater Sci 2006; 41: 7111-8.

[14] Asp LE. The effects of moisture and temperature on the interlaminar delamination toughness of a carbon/epoxy composite. Compos Sci Technol 1998; 58: 967-77.

[15] Ogi K, Kim HS, Maruyama T, Takao Y. The influence of hygrothermal conditions on the damage processes in quasiisotropic carbon/epoxy laminates. Compos Sci Technol 1999; 59: 2375-82.

[16] Garg A, Ishai O. Hygrothermal influence on delamination behavior of graphite/epoxy laminates. Eng Fract Mech 1985; 22: 413-27.

[17] Wiggenraad JFM, Zhang X, Davies GAO. Impact damage prediction and failure analysis of heavily loaded, blade-stiffened composite wing panels. Compos Struct 1999; 45: 81-103.
[18] Riccio A, Sellitto C, Gigliotti M. Influence of material uncertainty on the damage resistance and tolerance of stiffened composite panels. 16th ICCM International Conferences on Composite Materials proceedings; July 2007; Kyoto, Japan 2007.

[19] ANSYS MANUAL. Adv Analy Tech Guide (release 12). ANSYS INC 2009.

[20] Doucet A, de Freitas N, Gordon N, Eds. Sequential Monte Carlo methods in practice. New York: Springer 2001.

[21] Citarella R, Apicella A. Advanced design concepts and maintenance by integrated risk evaluation for aerostructures. Struct Durability Health Monit 2006; 2: 183-96.

[22] Lemieux C. Monte Carlo and Quasi-Monte Carlo sampling. New York: Springer 2009.

[23] Heltona JC, Davisb FJ, Johnsonc JD. A comparison of uncertainty and sensitivity analysis results obtained with random and Latin hypercube sampling. Reliab Eng Syst Saf 2005; 89: 305-30.

[24] Abrate S. Impact on composite structures. Cambridge Uni Press: Cambridge 2005.

(C) Riccio et al.; Licensee Bentham Open.

This is an open access article licensed under the terms of the Creative Commons Attribution Non-Commercial License (http://creativecommons.org/licenses/by-nc/3.0/) which permits unrestricted, non-commercial use, distribution and reproduction in any medium, provided the work is properly cited. 